\title{
PAGING INSPECTOR SANDS: THE COSTS OF PUBLIC INFORMATION Online Appendix
}

\author{
Sacha Kapoor and Arvind Magesan*
}

This appendix has two sections. In the first section we provide a simple theoretical example to rationalize the key empirical result from the paper, that the installation of countdown timers increased the propensity for collisions among drivers. In the second we provide discussion and evidence of the robustness of the key empirical findings to alternative estimation methods.

\section{A Textbook Example of Driver Interaction}

We modify a simple textbook model of driver interaction, ${ }^{1}$ where drivers can choose to act aggressively or cautiously, to rationalize the finding that informing drivers about light changes causes more driver-driver collisions. The modification is that we allow for uncertainty in what drivers know about the time until a light change at an intersection. We show that under rather innocuous assumptions, equilibrium collision probabilities are larger when drivers know the time that remains. The result follows from two simple features of equilibrium behavior in our model. First, aggressive behavior on the part of informed drivers is more likely when less time remains. Second, drivers are most responsive to information about light changes when they learn the time that remains is less than they expected. Taken together, the features of equilibrium behavior imply that informing drivers increases the chances of a collision.

\section{$1.1 \quad$ Model}

Suppose that two randomly matched drivers drawn from a single population approach an intersection from different directions. As they approach, each driver can choose either to proceed with caution $(C)$ or to act aggressively $(A)$. A driver who acts cautiously either slows down or stops, yielding the right-of-way to the other driver. A driver who acts aggressively either continues at the same speed or speeds up without conceding the right-of-way. We

\footnotetext{
*Kapoor: Erasmus University Rotterdam, Erasmus School of Economics H09-22, Burgemeester Oudlaan 50, 3062 PA Rotterdam, The Netherlands, kapoor@ese.eur.nl. Magesan: University of Calgary, Department of Economics, 2500 University Drive N.W., Calgary AB, T2N 1N4, Canada, anmagesa@ucalgary.ca.

${ }^{1}$ See Approaching Cars on page 130 of Osborne [2004].
} 
assume drivers are impatient and prefer the right-of-way to waiting for the other to pass. Drivers that are aggressive risk receiving a fine if they are caught, and risk collision with the other vehicle if its driver also chooses to be aggressive. This general framework encompasses several common interactions that occur at intersections. One such interaction occurs when a driver who is traveling straight through an intersection meets another who is turning left from the opposite direction. Another occurs when a driver is again traveling straight through but meets a driver who is turning right from the adjacent street.

Let $v>0$ be the payoff from obtaining the right-of-way, $c>0$ the cost of collision, and $b>0$ be the cost of a fine to a driver caught crossing the intersection when the light is red. We assume that $c>b$. Let $p$ be the probability that a collision occurs when both drivers act aggressively. Let $P_{T}(\omega)$ be the probability that a driver is caught and fined when he acts aggressively. $\omega$ is a random variable that represents the number of seconds until a light change (from green to yellow). Its probability distribution is given by $F(\omega)$. We assume that $P_{T}(\omega)$ decreases as $\omega$ increases. That is, the probability of a fine when acting aggressively diminishes as the amount of time left before the light changes from green to yellow increases. For example, driving through a red light is more likely when the driver acts aggressively just before a light change than when he acts aggressively with plenty of time remaining.

$\omega$ is known to drivers when a countdown is present at the intersection, and unknown to drivers when there is no countdown. When $\omega$ is known, drivers have better information about the consequences of conceding the right-of-way to another road user. In particular, drivers know whether conceding the right-of-way will cause them to have to sit through a red light.

The normal form for the simple game we consider is presented in Figure I, where one driver chooses a row and the other a column. Payoffs are symmetric. The matrix lists the payoffs for the row player. $\pi(\omega)$ is the payoff to acting aggressively when the other driver

\begin{tabular}{|c|c|c|}
\hline \multirow{3}{*}{$\begin{array}{l}\text { Cautious } \\
\text { Aggressive }\end{array}$} & Cautious & Aggressive \\
\hline & 0 & 0 \\
\hline & $\pi(\omega)$ & $p[-c]+(1-p) \pi(\omega)$ \\
\hline
\end{tabular}

Figure I: The game when countdowns are active.

acts cautiously, where

$$
\pi(\omega)=P_{T}(\omega)[-b]+\left(1-P_{T}(\omega)\right) v
$$

We assume that the chances of getting caught and fined for aggressive behavior is small enough so that $\pi(\omega) \geq 0$. If $\pi(\omega)<0$, being cautions is the dominant strategy for both players, and in equilibrium collisions are never observed.

\subsection{Public Information Increases Collision Probabilities}

Under these assumptions, the game has three Nash equilibria: two asymmetric pure strategy Nash equilibria where one driver is cautious and the other aggressive and a symmetric mixed 
strategy Nash equilibrium (MSNE) where each player acts cautiously with probability

$$
q^{*}(\omega)=\frac{p c-(1-p) \pi(\omega)}{p(c+\pi(\omega))} .
$$

We focus our analysis on the MSNE for two important reasons. The first is that pure strategy equilibria are at odds with what we observe in the data, as they suggest that collisions never happen. On the other hand, when drivers use mixed strategies, the equilibrium probability that an collision occurs is given by:

$$
\begin{aligned}
P_{i}^{*}(a \mid \omega) & =\left(1-q^{*}(\omega)\right)^{2} p \\
& =\frac{1}{p}\left[\frac{\pi(\omega)}{c+\pi(\omega)}\right]^{2}>0 .
\end{aligned}
$$

The second reason we focus on MSNE is that the MSNE is the only symmetric Nash equilibrium of the game. ${ }^{2}$ The pure strategy equilibria each require one driver to defer to the other by social convention. However, we are unaware of any social convention that would lead one of these equilibria to be the norm. ${ }^{3}$

\begin{tabular}{|c|c|c|}
\hline & Cautious & Aggressive \\
\hline Cautious & 0 & 0 \\
\hline Aggressive & $E \pi(\omega)$ & $p[-c]+(1-p) E \pi(\omega)$ \\
\hline
\end{tabular}

Figure II describes the game where drivers are unable to observe countdown signals and are therefore uninformed about the time until a light change. $E \pi(\omega)$ is the expected payoff

Figure II: The game when countdowns are inactive.

to acting aggressively, where the expectation is taken with respect to $\omega .{ }^{4}$ Similar to the case where drivers are informed about $\omega$, the unique MSNE probability of being cautious is given by:

$$
q^{*}(F)=\frac{p c-(1-p) E \pi(\omega)}{p(c+E \pi(\omega))} .
$$

We can use the unique MSNE to solve for the collision probability when there are no countdown timers - drivers are uninformed:

$$
\begin{aligned}
P^{*}(a \mid F) & =\left(1-q^{*}(F)\right)^{2} p \\
& =\frac{1}{p}\left[\frac{E \pi(\omega)}{c+E \pi(\omega)}\right]^{2} .
\end{aligned}
$$

\footnotetext{
${ }^{2}$ In the language of evolutionary game theory, the MSNE is the unique evolutionarily stable strategy of our game.

${ }^{3}$ As we imagine that drivers are drawn and matched randomly from the same population, the MSNE can also be interpreted as the 'steady state' of interactions at intersections. Under this interpretation some fraction of the population of drivers act cautiously while the other fraction acts aggressively (See pp.37-39 of Osborne and Rubinstein [1994]).

${ }^{4}$ We assume that driver beliefs about the time until a light change are consistent with the true distribution $F(\omega)$.
} 
Proposition 1 The probability of an accident occurring at an intersection is larger when countdowns are present than when they are not:

$$
E_{\omega}\left[P^{*}(a \mid \omega)\right]>P^{*}(a \mid F)
$$

Proof 1 Simple algebra reveals that:

$$
E_{\omega}\left[P_{i}^{*}(a \mid \omega)\right]>P^{*}(a \mid F) \Leftrightarrow E\left[\frac{1}{1-\frac{-c}{\pi(\omega)}}\right]^{2}>\left[\frac{1}{1-\frac{-c}{E \pi(\omega)}}\right]^{2} .
$$

Our assumption that $c>b$ combined with Jensen's inequality ensure that $E\left[\frac{1}{1-\frac{-c}{\pi(\omega)}}\right]^{2}>$ $\left[\frac{1}{1-\frac{-c}{E \pi(\omega)}}\right]^{2}$. To see this Let $h(r)=\frac{\pi(\omega)}{c+\pi(\omega)}$ where $r=P_{T}(\omega)$. We define $f(t) \equiv \frac{1}{(1-t)}$ and $g(r) \equiv \frac{c}{r(b+v)-v}$ so that $h(r)=f(g(r))$. The functions $f$ and $g$ have the following properties: a) $f(t)$ is increasing and convex if $t<1 ; b) g$ is decreasing and convex in $r$. These properties imply that $h$ is convex in $r$ for $t<1$. Or, equivalently, $h(r)$ is convex in $r$ if and only if $-c<r(-b)+(1-r) v$. The inequality $-c<r(-b)+(1-r) v$ clearly holds when $c>b$. It follows that the square $h(r)^{2}$ is also convex in $r$. Since $r$ is a monotone function of $\omega$, applying Jensen's Inequality to $h(r)^{2}$ yields the result.

Mathematically speaking, the result is driven by the fact that the MSNE probability a driver is aggressive is convex and increasing in $\omega$.

Consider figure III, which illustrates the key properties of the equilibrium with and without knowledge of $\omega$. In the figure $q(\omega)$ traces the informed driver's equilibrium probability of behaving aggressively as a function of the state of the world $\omega . q(F)$ represents the uninformed driver's equilibrium probability of behaving aggressively. It is independent of the true state of the world. $\Delta$ represents a unit change in the time remaining until a light change. $\omega_{0}$ satisfies $q\left(\omega_{0}\right)=q(F)$. Two important facts are evident in the figure:

1. As $\omega$ gets smaller informed drivers are more likely to be aggressive - $q(\omega)$ is decreasing.

2. The sensitivity of a driver's behavior to knowledge of $\omega$, as measured by $q(\omega)-q(F)$, is largest when the information is that he has less time than he expected.

Taken together, the facts imply that the presence of countdown timers raises the propensity for collision. Driver behavior is most responsive to information about the state of the world, $\omega$, precisely at the states of the world where they are most aggressive. As such, on average, collisions are more likely when a countdown timer is present at the intersection. 
Figure III: Equilibrium Probability of Aggression with and without Knowledge of $\omega$

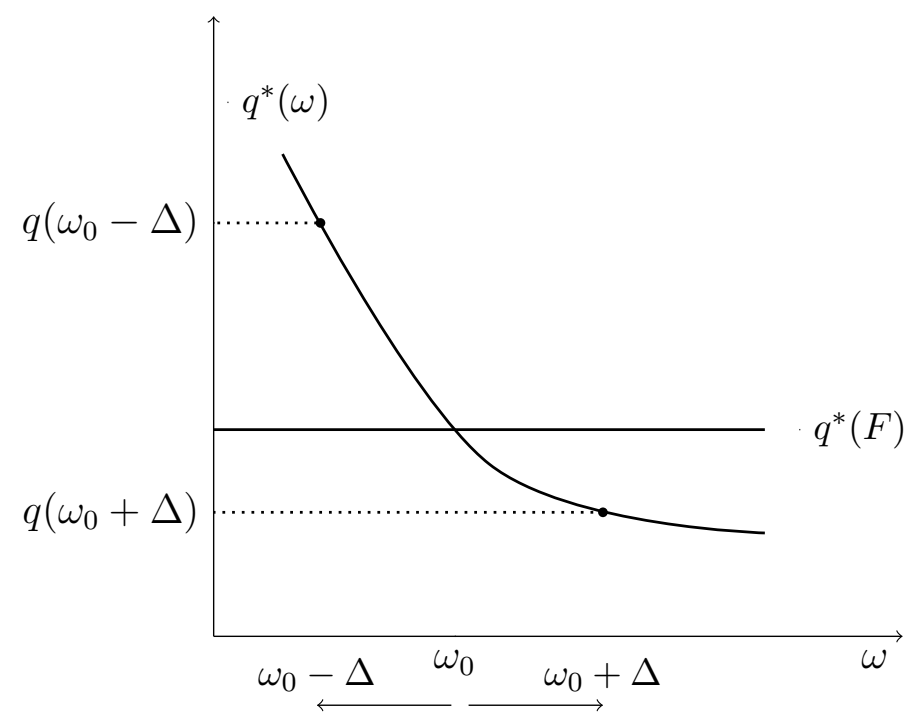

\section{Robustness}

The outcome variable in our study is the number of collisions, which is of course a nonnegative integer, or more specifically, a count variable. As such, one may argue that count data methods are more appropriate than Ordinary Least Squares with Fixed Effects (OLS FE) for estimating the effect of interest in our study. Unlike the case of OLS, the estimating equation in the count data context is non-linear in nature. Thus, while the fixed effects are simply differenced away in the OLS FE case, they can not simply be differenced away in these other non linear frameworks. Estimating the fixed effects may introduce an incidental parameters problem and lead to unreliable estimates of the effect of interest. Not including fixed effects is not a viable option either, as a key part of our identification strategy is being able to control for permanent, unobserved differences across intersections.

Hausman et al. [1984] propose a method for estimating count models while allowing for permanent unobserved heterogeneity. They show that under certain assumptions about the structure of the econometric error, conditioning on the sum (over time) of the outcome for each intersection delivers a likelihood function that does not depend on intersection fixed effects. An implication of the Hausman et al. [1984] approach, however, is that it excludes intersections where there were no collisions. Because the number of collisions is a nonnegative integer, if the sum over time is zero then the count in any particular month must also be zero. It follows that the collision probability in any particular month is zero and, consequently, that observations from these intersections cannot contribute to the likelihood function. This is a serious issue in our context for two reasons. First, the intersections that are dropped are not randomly dropped. Put differently, intersections where countdowns were installed and where there were no collisions are informative for accurately estimating 
average treatment effects. Restricting the analysis to the selected sample where at least one collision occurs yields an overestimate of the average effect. The second reason is that there is a substantial loss in statistical power when we limit the analysis to the selected sample. The loss of statistical power is particularly important in the present context because we are studying the effects of countdown timers on a rare event (collisions). With rare events, one requires an abundance of data in order to detect seemingly small yet statistically significant effects. As the [Hausman et al., 1984] method excludes 819 intersections, or approximately 46 percent of the intersections in the sample, the loss of power in our context is particularly severe.

Table I illustrates the problems that come from restricting the sample to a non-random sample of intersections experiencing at least one collision. Column 4 of Table I presents the marginal effect from a Poisson regression (employing the Hausman et al. [1984] method) of collisions on the introduction of a countdown. The marginal effect implies the increase in collisions for the average intersection in the restricted sample was 17 percent. Unsurprisingly, this effect is much larger than the 5 percent estimate implied by OLS fixed estimates that use a representative sample (Column 1). It is also unsurprising that the estimate is statistically insignificant - there is substantial loss in statistical power that comes from basing the estimate on the restricted sample of 975 intersections rather than on the representative sample of 1794 intersections.

Column 2 of Table I presents OLS FE estimates of the effect of countdowns for the restricted sample the Hausman et al. [1984] method uses. The estimate further illustrates the effects of selection and a loss in statistical power - it shows that this effect is not specific to the Hausman et al. [1984] method. At the same time the coefficient estimate is more conservative than the Poisson fixed effects estimate of Column 4. The estimate implies the introduction of a countdown resulted in 0.019 more accidents at the average intersection, where the estimate is statistically significant at the 10 percent level. As with the Poisson estimate, the estimate of column 2 is much larger than the estimate of Column 1 . It is also more imprecisely estimated, as its standard error is more than double the standard error of Column 1. The estimate is more conservative than the Poisson estimate, as it translates into an 8.3 percent increase (rather than a 17 percent increase) in the number of collisions after a countdown was introduced.

Ideally, to deal with the fact that collisions are rare, one would like to apply "zeroinflated" count methods to the full sample [Cameron and Trivedi, 2005]. Zero-inflated methods account for rare events by explicitly modeling a separate data generating process for the zeros in the sample. Unfortunately, these methods not yet developed to the point where they can account for fixed effects. This is a problem because, as the OLS FE estimates for the full sample reveal, accounting for the fixed effects is crucial for properly evaluating the effects of countdown timers. It shows, specifically, the importance of using the substantial within-intersection variation in identifying a treatment effect.

Since there is no well-developed statistical framework for accounting for both fixed effects and counts with excess zeros, we provide a back-of-the-envelope Poisson estimate that allows us to keep all of intersections in the data while, at the same time, accounting for fixed effects. 
Table I: Poisson with Fixed Effects, Selection, and a Loss in Statistical Power

\begin{tabular}{l|cccc}
\hline & $\begin{array}{c}\text { OLS FE on } \\
\text { Representative } \\
\text { Sample }\end{array}$ & $\begin{array}{c}\text { OLS FE on } \\
\text { Selected } \\
\text { Sample }\end{array}$ & $\begin{array}{c}\text { Back-of-the } \\
\text { Envelope } \\
\text { Poisson FE on } \\
\text { Representative } \\
\text { Sample }\end{array}$ & $\begin{array}{c}\text { Poisson FE on } \\
\text { Selected } \\
\text { Sample }\end{array}$ \\
\hline \hline Pedestrian Countdown & $0.012^{* *}$ & $0.019^{*}$ & $0.012^{* *}$ & 0.040 \\
Signal Activated & $(0.006)$ & $(0.012)$ & $(0.006)$ & $(0.040)$ \\
\hline $\begin{array}{l}\text { Implied Percentage } \\
\text { Change in Collisions }\end{array}$ & 5.2 & 8.3 & 5.2 & 17.5 \\
\hline Intersections & 1794 & 975 & 1794 & 975 \\
Observations & 107640 & 58500 & 107640 & 58500 \\
\hline
\end{tabular}

1. The dependent variable is number of collisions.

2. Robust Standard Errors clustered at the intersection level, *** for $p<.01$, ** for $.01<p<.05,{ }^{*}$ for $p<.1$.

3. Regressions include intersection and month-year fixed effects.

The approach proceeds in two-steps. In the first step we use OLS to "partial out" the intersection and time fixed effects from our treatment variable, by regressing the treatment variable on time and intersection fixed effects and retaining the residual. In the second step we simply run a standard Poisson regression of accident count on this residual. ${ }^{5}$ We do not need to consider fixed effects in this second stage because the treatment residual is by definition orthogonal to the fixed effects. It is the part of the treatment not explained by time and intersection fixed effects. This back-of-the-envelope estimate is found in Column 3 of Table I. Note the striking similarity to the result in Column 1, where the OLS FE estimator (for the full sample) is presented. The average partial effect is 0.012 , an estimate which implies a 5.2 percent increase in collisions at a representative intersection after the countdown timer is introduced. The average partial effect, its statistical significance, and implied percentage change are all similar to the one we obtain with OLS FE on the full sample.

While the lack of a feasible count data method is disappointing in our context, OLS estimation of econometric models with a non-negative integer outcome is not necessarily problematic in terms of obtaining consistent estimates of the effect of interest. OLS regression applied to a model with a binary outcome (the Linear Probability Model) "produces consistent and even unbiased estimators" of the regression coefficients [Wooldridge, 2010, pg. 562] and in the case of models where the outcome is a count, OLS may "provide good estimates of average partial effects (APE's) on the conditional mean." [Wooldridge, 2010, pg. 723] The real drawback of applying OLS to econometric models with a non-negative integer

\footnotetext{
${ }^{5}$ Note that if we were to consider simple OLS regression of accident count on the residual in the second stage, by the Frisch-Waugh Theorem we would simply obtain the same estimate as in Column 1 (the main result of the paper) [Angrist and Pischke, 2009, pg. 35].
} 
Table II: Collisions and Countdowns with Bootstrapped Standard Errors

\begin{tabular}{l|cccc}
\hline & $(1)$ & $(2)$ & $(3)$ & $(4)$ \\
\hline \hline \multirow{2}{*}{$\begin{array}{l}\text { Pedestrian Countdown } \\
\text { Signal Activated }\end{array}$} & $\begin{array}{c}-0.055^{* * *} \\
(0.005)\end{array}$ & $\begin{array}{c}-0.022^{* * *} \\
(0.004)\end{array}$ & $\begin{array}{c}0.012^{* * *} \\
(0.004)\end{array}$ & $\begin{array}{c}0.011^{* *} \\
(0.004)\end{array}$ \\
\cline { 1 - 4 } Controls & $\mathrm{N}$ & $\mathrm{Y}$ & $\mathrm{Y}$ & $\mathrm{Y}$ \\
\cline { 1 - 2 } Intersection & $\mathrm{N}$ & $\mathrm{N}$ & $\mathrm{Y}$ & $\mathrm{Y}$ \\
Month-Year & $\mathrm{N}$ & $\mathrm{N}$ & $\mathrm{N}$ & $\mathrm{Y}$ \\
Lagged Collisions & 1794 & 1794 & 1794 & 1794 \\
\cline { 1 - 2 } Intersections & 107640 & 107640 & 107640 & 105846 \\
\cline { 1 - 2 } Observations &
\end{tabular}

1. The dependent variable is number of collisions.

2. Bootstrap Standard Errors (based on 10 replications) in parentheses, *** for $p<.01,{ }^{* *}$ for $.01<p<.05, *$ for $p<.1$.

outcome is that the usual OLS test statistics do not apply. Essentially, the count nature of the outcome implies that the statistical error is not normally distributed. However, normality of the error term in a regression is not a prerequisite for consistency or unbiasedness of the OLS estimator. Normality of the error term is typically assumed in when using OLS in order to ensure that the test statistic has a t-distribution. In other words, if the errors of the regression model are not normally distributed, it is not necessarily the case that the usual critical values apply. That is, the count nature of the outcome is a problem for inference, not identification.

We tackle the inference issue in a couple ways. First, we consider the most common alternative method for approximating standard errors, bootstrapping. The validity of bootstrapped standard errors and the resulting test statistics does not rely on the assumption of normality of the regression model error.

Table II presents the coefficient estimates from our main table (Table 2 in the paper) with bootstrap standard errors in place of the usual standard errors. The results show that bootstrapping the standard errors leads to more precise estimates of the effect of countdowns on accidents. Specifically, Column 3 of Table II shows the $p$-value falls from a value less than 0.05 to a value less than 0.01 . Thus the main result of the paper is robust to relaxing the assumption of a normal error.

Second, we consider a simple transformation of our outcome variable to a continuous measure. We redefine the outcome to be the ratio of number of accidents to total traffic flow through an intersection. We thus consider the effect of countdowns on collisions per-capita, or the rate of collisions, a smooth variable. In principle, the collision rate can equal any positive real number. As such, it is more likely that the error in a regression model follows a normal distribution than when the dependent variable is a (discrete) count. Fixed effect estimates with the accident rate as the dependent variable are found in Table III. The estimates show our main results are robust to our continuous transformation of the dependent variable. 
Table III: The Effect of Countdowns on Collision Rates

\begin{tabular}{l|cccc}
\hline & $(1)$ & $(2)$ & $(3)$ & $(4)$ \\
\hline \hline \multirow{2}{*}{$\begin{array}{l}\text { Pedestrian Countdown } \\
\text { Signal Activated }\end{array}$} & $-0.004^{* *}$ & $-0.002^{* *}$ & $0.00093^{*}$ & $0.00095^{*}$ \\
\cline { 1 - 4 } Controls & $\mathrm{N}$ & $\mathrm{Y}$ & $\mathrm{Y}$ & $\mathrm{Y}$ \\
\cline { 1 - 2 } Intersection & $\mathrm{N}$ & $\mathrm{N}$ & $\mathrm{Y}$ & $\mathrm{Y}$ \\
Month-Year & $\mathrm{N}$ & $\mathrm{N}$ & $\mathrm{N}$ & $\mathrm{Y}$ \\
Lagged Collisions & 1794 & 1794 & 1794 & 1794 \\
\cline { 1 - 2 } Intersections & 106393 & 106393 & 106393 & 105552 \\
Observations &
\end{tabular}

1. The dependent variable is number of collisions.

2. Robust Standard Errors clustered at the intersection level, *** for $p<.01,{ }^{* *}$ for $.01<p<.05, *$ for $p<.1$.

Finally, we end the robustness section by illustrating that our main result is robust to the inclusion of several lags of the dependent variable. Estimates with two to six lags are found is Table IV. The table shows there were 0.010 more collisions at the average intersection once a countdown was introduced, where the estimates are generally statistically significant at the 10 percent level. As Table IV illustrates, the point estimates and statistical significance are strikingly similar across all five specifications. ${ }^{6}$

\footnotetext{
${ }^{6}$ Two factors can explain the decline in statistical significance in Column 6 . The first is that the statistical power of our estimator falls as we exchange more lags for fewer observations. The decline in statistical power makes it more difficult to detect small but statistically significant effects. The second is that the incidental parameters problem, which arises because autoregressive parameters mechanically depend on intersection fixed effects, is of greater concern as the cross-sectional dimension grows relative to the time-series dimension. This dimensionality problem reduces the chances of obtaining consistent estimates of the countdown's effect.
} 
Table IV: Collisions and Pedestrian Countdown Signals

\begin{tabular}{l|ccccc}
\hline & $(1)$ & $(2)$ & $(3)$ & $(4)$ & $(5)$ \\
\hline \hline \multirow{2}{*}{$\begin{array}{l}\text { Pedestrian Countdown } \\
\text { Signal Activated }\end{array}$} & $\begin{array}{c}0.010^{*} \\
(0.006)\end{array}$ & $\begin{array}{c}0.010^{*} \\
(0.006)\end{array}$ & $\begin{array}{c}0.010^{*} \\
(0.006)\end{array}$ & $\begin{array}{c}0.010^{*} \\
(0.006)\end{array}$ & $\begin{array}{c}0.010 \\
(0.006)\end{array}$ \\
\cline { 1 - 4 } Controls & \multirow{2}{*}{ Two } & Three & Four & Five & Six \\
\cline { 1 - 4 } Collision Lags & 1794 & 1794 & 1794 & 1794 & 1794 \\
Intersections & 104052 & 102258 & 100464 & 98670 & 96876 \\
\hline
\end{tabular}

1. The dependent variable is number of collisions.

2. Robust Standard Errors clustered at the intersection level, *** for $p<.01,{ }^{* *}$ for $.01<p<.05, *$ for $p<.1$.

3. Regressions include intersection and time fixed effects as well as a control for the months since the first installation. 


\section{References}

Joshua D. Angrist and Jorn-Steffen Pischke. Mostly Harmless Econometrics. Princeton University Press, Princeton, New Jersey, 2009.

A. Colin Cameron and Pravin K. Trivedi. Microeconometrics: Methods and Applications. Cambridge University Press, New York, May 2005.

Jerry Hausman, Bronwyn H. Hall, and Zvi Griliches. Econometric models for count data with an application to the patents-r and d relationship. Econometrica, 52(4):909-938, July 1984.

Martin J. Osborne. An Introduction to Game Theory. Oxford University Press, 198 Madison Avenue, New York, New York 10016, 2004.

Martin J. Osborne and Ariel Rubinstein. A Course in Game Theory. The MIT Press, Cambridge, Massachusetts and London, England, 1994.

Jeffrey M Wooldridge. Econometric Analysis of Cross Section and Panel Data, volume 1. The MIT Press, 2nd edition, 2010. 